\title{
On irregularities in the graph of generalized divisor functions
}

\author{
by \\ Gergely ZÁBrÁdi (Budapest)
}

1. Introduction. It is partly known [1], partly easy to prove that for the divisor function

$$
d(n):=\sum_{d \mid n} 1,
$$

it is true that for all $\omega>0$ there is an $n \in \mathbb{N}$ such that

$$
d(n)>\omega+\max (d(n-1), d(n+1))
$$

and also there is an $m \in \mathbb{N}$ such that

$$
d(m)+\omega<\min (d(m-1), d(m+1)) .
$$

P. Erdős [1] proved (2) in the following stronger form: for all $k \in \mathbb{N}$ there are infinitely many $n \in \mathbb{N}$ such that

$$
d(n)>\prod_{i=1}^{k} d(n-i) d(n+i) .
$$

We will extend these theorems to generalized divisor functions $d(\mathcal{A}, n)$ defined for any set $\mathcal{A} \subseteq \mathbb{N}$ as

$$
d(\mathcal{A}, n):=\sum_{a \in \mathcal{A}, a \mid n} 1 .
$$

These functions were introduced by Erdős and Sárközy [2]. Among other results they proved that for any infinite $\mathcal{A}$ the large values of $d(\mathcal{A}, n)$ are much greater than its average:

$$
\limsup _{N \rightarrow \infty} \frac{\max _{n \leq N} d(\mathcal{A}, n)}{\sum_{a \in \mathcal{A}, a \leq N} 1 / a}=\infty .
$$

A. Sárközy posed the following three related problems in [5] (Problems 25-27):

2000 Mathematics Subject Classification: Primary 11N36. 
Problem 1. Is it true that $|d(\mathcal{A}, n+1)-d(\mathcal{A}, n)|$ cannot be bounded for an infinite set $\mathcal{A} \subseteq \mathbb{N}$ ?

Problem 2. Is it true that for any infinite set $\mathcal{A} \subseteq \mathbb{N}$ there are infinitely many $n$ with

$$
d(\mathcal{A}, n)>\max (d(\mathcal{A}, n+1), d(\mathcal{A}, n-1)) ?
$$

Problem 3. What assumption is needed to ensure that

$$
d(\mathcal{A}, n)<\min (d(\mathcal{A}, n-1), d(\mathcal{A}, n+1))
$$

for infinitely many $n$ ?

This article solves these problems and also generalizes Erdős's theorem.

2. Notation and the lemma. Following [4], we will use the following notations: Let $\mathcal{B} \subset \mathbb{N}$ be an arbitrary finite sequence, $X:=|\mathcal{B}|$. Let $\mathcal{P} \subset \mathbb{N}$ be an arbitrary set of primes. Set

$$
\begin{aligned}
P(z) & :=\prod_{p \in \mathcal{P}, p \leq z} p . \\
S(\mathcal{B}, \mathcal{P}, z) & :=|\{b: b \in \mathcal{B},(b, P(z))=1\}| .
\end{aligned}
$$

Let $\omega$ be a multiplicative arithmetical function such that $\omega(n)=0$ if $n$ is not squarefree and also if $n$ has a prime factor not in $\mathcal{P}$, and $\omega(1):=1$. Let $\gamma$ be Euler's constant and $\Gamma$ be the well-known Gamma function, $\mu$ be the Möbius function, and $\nu(d)$ be the number of distinct prime divisors of $d$. We define

$$
\begin{gathered}
W(z):=\prod_{p \leq z}\left(1-\frac{\omega(p)}{p}\right) . \\
\sigma_{\kappa}(u):=2^{-\kappa} \frac{e^{-\gamma \kappa}}{\Gamma(\kappa+1)} u^{\kappa} \quad \text { if } 0 \leq u \leq 2, \\
\left(u^{-\kappa} \sigma_{\kappa}(u)\right)^{\prime}:=-\kappa u^{-\kappa-1} \sigma_{\kappa}(u-2) \quad \text { if } u>2,
\end{gathered}
$$

with $\sigma_{\kappa}$ required to be continuous at $u=2$. We set

$$
\begin{gathered}
\eta_{\kappa}(u):=\kappa u^{-\kappa} \int_{u}^{\infty} t^{\kappa-1}\left(\frac{1}{\sigma_{\kappa}(t-1)}-1\right) d t \quad(u>1) . \\
R_{d}:=|\{b \in \mathcal{B}: d \mid b\}|-\frac{\omega(d)}{d} X \quad \text { if } \mu(d) \neq 0 .
\end{gathered}
$$

Let us now define four properties as in [4]:

$\left(\Omega_{1}\right)$ : There exists $A_{1}$ such that $0 \leq \omega(p) / p \leq 1-1 / A_{1}$ for all primes $p$. $\left(\Omega_{2}\left(\kappa, A_{2}, A_{3}\right)\right)$ : There exist $\kappa \geq 0$ and $A_{2}, A_{3} \geq 1$ such that 


$$
-A_{2} \leq \sum_{w \leq p<z \text { prime }} \frac{\omega(p) \log p}{p}-\kappa \log \frac{z}{w} \leq A_{3} \quad \text { if } 2 \leq w \leq z .
$$

(R): $\left|R_{d}\right| \leq \omega(d)$ if $\mu(d) \neq 0$, and $(d, p)=1$ for all $p \notin \mathcal{P}$.

$(\mathrm{R}(\kappa, \alpha))$ : There exist constants $0<\alpha<1$ and $A_{4}, A_{5} \geq 1$ such that if $X \geq 2$ then

$$
\sum_{\substack{d<X^{\alpha} /(\log X)^{A_{4}} \\ \forall p \notin \mathcal{P}(d, p)=1}} \mu^{2}(d) 3^{\nu(d)}\left|R_{d}\right| \leq A_{5} \frac{X}{\log ^{\kappa+1} X} .
$$

It is not difficult to see that $(\mathrm{R}(\kappa, \alpha))$ is less restrictive than $(\mathrm{R})$ beside $\left(\Omega_{1}\right)$ (see [4]). The strongest lower bound for $S(\mathcal{B}, \mathcal{P}, z)$ in $[4]$ is the following:

Lemma 1 (see [4, p. 219]). If $\left(\Omega_{1}\right),\left(\Omega_{2}\left(\kappa, A_{2}, A_{3}\right)\right)$ and $(\mathrm{R}(\kappa, \alpha))$ hold and

$$
z^{2} \leq X^{\alpha} /(\log X)^{A_{4}} \quad(X \geq 2)
$$

then

$$
S(\mathcal{B}, \mathcal{P}, z) \geq X W(z)\left(1-\eta_{\kappa}\left(\alpha \frac{\log X}{\log z}\right)-A_{6} \frac{A_{2}(\log \log 3 X)^{3 \kappa+2}}{\log X}\right)
$$

where $A_{6} \geq 1$ is a constant which depends only on $\kappa, \alpha, A_{1}, A_{2}, A_{3}, A_{4}, A_{5}$.

\section{The results}

Theorem 1. Let $\mathcal{A}=\left\{a_{1}<a_{2}<\ldots\right\} \subseteq \mathbb{N}$ and $k \in \mathbb{N}$. Then there exist infinitely many $n \in \mathbb{N}$ such that

$$
d(\mathcal{A}, n)>\prod_{i=1}^{k} d(\mathcal{A}, n-i) d(\mathcal{A}, n+i) .
$$

Proof. We are going to prove that there exists a constant $C=C(k)>0$ such that there are infinitely many $n$ for which

$$
\prod_{i=1}^{k} d(\mathcal{A}, n-i) d(\mathcal{A}, n+i)<C
$$

and $d(\mathcal{A}, n)$ can be arbitrarily large for these $n$ 's. Define

$$
\begin{aligned}
X & :=\prod_{p \leq 2 k+1 \text { prime }} p^{1+\left[\log _{p} k\right]} \prod_{j=1}^{N} a_{j}, \\
\mathcal{B} & :=\left\{\prod_{i=1}^{k}(j X-i)(j X+i): j \in\{1, \ldots, X\}\right\}, \\
\mathcal{P} & :=\{p:(p, X)=1 \text { prime }\}, \\
\omega(p) & :=2 k \quad \text { if } p \in \mathcal{P},
\end{aligned}
$$


and extend $\omega$ multiplicatively to squarefree $d$ 's for which $(d, p)=1$ if $p \notin \mathcal{P}$. It is easy to see that $|\mathcal{B}|=X$. Now we should check the conditions we need for the lemma:

$\left(\Omega_{1}\right)$ : Since $0 \leq \omega(p) \leq 2 k$ and $p>2 k+1$ if $\omega(p) \neq 0$, we have

$$
0 \leq \frac{\omega(p)}{p} \leq 1-\frac{1}{2 k+1} .
$$

$\left(\Omega_{2}\left(\kappa, A_{2}, A_{3}\right)\right)$ : This condition is trivial by the following well-known statement:

$$
\sum_{w \leq p<z \text { prime }} \frac{\log p}{p}=\log \left(\frac{z}{w}\right)+O(1) \quad \text { if } 2 \leq w \leq z
$$

because $0 \leq \omega(p) \leq 2 k$, and $\omega(p)=2 k$ if $p>2 k+1$.

$(\mathrm{R}(\kappa, \alpha))$ : It is enough to prove ( $\mathrm{R})$ because it is more restrictive beside $\left(\Omega_{1}\right)$. Suppose that $d=\prod_{r=1}^{l} p_{r}$ where $p_{r} \in \mathcal{P}$ are distinct primes. We can get $|\{b \in \mathcal{B}: d \mid b\}|$ by counting how many $j \in\{1, \ldots, X\}$ there exist such that $p_{r} \mid j X+i_{r}$ for fixed $i_{r} \in\{1, \ldots, k,-1,-2, \ldots,-k\}$ for all $1 \leq r \leq l$. Now $(X, d)=1$ and this condition holds for $j$ if and only if it does for $j+d$, so there are $[X / d]$ or $[X / d]+1$ pieces of such $j$ 's. Hence if we take it $X / d$ then the bias is at most 1 . There are $(2 k)^{l}=\omega(d)$ choices for the $i_{r}$ 's and therefore $\left|R_{d}\right| \leq \omega(d)$.

Now we can use the lemma. Let $z=X^{1 / c}$ and choose $c$ such that

$$
\begin{gathered}
z^{2} \leq \frac{X^{\alpha}}{(\log X)^{A_{4}}}, \\
\eta_{\kappa}\left(\alpha \frac{\log X}{\log z}\right)=\eta_{\kappa}(\alpha c)<1
\end{gathered}
$$

for $X$ large enough. Such a $c$ exists because $\eta_{\kappa}$ is a decreasing function with limit 0 at $+\infty$. Now we choose $N$ large enough and

$$
N>\left(2^{4 k c} \prod_{p \leq k \text { prime }}\left(2[k / p]\left[\log _{p} k\right]+1\right)\right)^{2 k} .
$$

Then

$$
1-\eta_{\kappa}\left(\alpha \frac{\log X}{\log z}\right)-A_{6} \frac{A_{2}(\log \log 3 X)^{3 \kappa+2}}{\log X}>0 .
$$

So we can conclude from the lemma that $S(\mathcal{B}, \mathcal{P}, z)>0$, which means that there exists $b \in \mathcal{B}$ with $(b, p)=1$ if $p \in \mathcal{P}$ and $p \leq z$, and $b=$ $\prod_{i=1}^{k}(j X-i)(j X+i)$ for some $j \in\{1, \ldots, X\}$. In view of the lemma below, $n=j X$ is a good choice for the theorem.

LEMma 2. We have

$$
d(\mathcal{A}, j X \pm i) \leq d(\mathcal{A}, b) \leq d(b) \leq 2^{4 k c} \prod_{p \leq k \text { prime }}\left(2[k / p]\left[\log _{p} k\right]+1\right) .
$$


Proof. The first two inequalities are trivial. For the third one we use the formula $d\left(\prod_{i=1}^{m} p_{i}^{\alpha_{i}}\right)=\prod_{i=1}^{m}\left(\alpha_{i}+1\right)$ :

1. If $p \leq k$ then $p^{1+\left[\log _{p} k\right]} \mid X$ so only $2[k / p]$ factors in

$$
b=\prod_{i=1}^{k}(j X-i)(j X+i)
$$

are divisible by $p$ and all of them contain at most $\left[\log _{p} k\right]$ factors $p$ because $p^{1+\left[\log _{p} k\right]}>k$.

2. If $k<p$ and $p \mid X$ then $(p, b)=1$.

3. If $k<p$ and $(p, X)=1$ then $p \in \mathcal{P}$. So if $p \leq z$ then $(p, b)=1$ else these primes give at most a multiplier of $2^{4 k c}$ in $d(b)$ because $b<X^{4 k}=$ $z^{4 k c} \leq p^{4 k c}$.

Now the proof of the theorem can be completed: For $n=j X$,

$$
\begin{aligned}
d(\mathcal{A}, n) & \geq N>\left(2^{4 k c} \prod_{p \leq k \text { prime }}\left(2[k / p]\left[\log _{p} k\right]+1\right)\right)^{2 k} \\
& \geq \prod_{i=1}^{k} d(\mathcal{A}, n-i) d(\mathcal{A}, n+i) .
\end{aligned}
$$

From this theorem we know that the generalized divisor functions have isolated large values. One may ask: what about the isolated small values? The set $\mathcal{A}=\{a: a \in \mathbb{N}, 3 \mid a\}$ shows that it may occur that

$$
d(\mathcal{A}, n)<\min (d(\mathcal{A}, n-1), d(\mathcal{A}, n+1))
$$

never holds. The following two theorems answer the question by giving a necessary and sufficient condition on $\mathcal{A}$.

TheOREM 2. There are infinitely many $n \in \mathbb{N}$ such that

$$
d(\mathcal{A}, n)<\min (d(\mathcal{A}, n-1), d(\mathcal{A}, n+1))
$$

if and only if there exist $a, b \in \mathcal{A}$ (not necessarily distinct) such that $a, b>1$ and $(a, b) \leq 2$.

Proof. One direction is trivial because if there exists an $n \in \mathbb{N}$ such that (27) holds then $n-1$ and also $n+1$ must have a divisor in $\mathcal{A}$; the two divisors are greater than 1 and their greatest common divisor is at most 2 .

For the other direction assume that $a, b \in \mathcal{A}$ are such that $a, b>1$ and $(a, b) \leq 2$. From the Chinese Remainder Theorem we know that there is a residue-class $\bmod [a, b]$ which is congruent to $1(\bmod a)$ and $-1(\bmod b)$. From Dirichlet's theorem we see that there are infinitely many prime numbers in this residue-class. If infinitely many of these primes do not belong to $\mathcal{A}$ then we are done. If all but finitely many of these primes belong to $\mathcal{A}$ then let $p_{1}<p_{2}<p_{3}<p_{4}$ be such primes from the set $\mathcal{A}$. 
Applying again the Chinese Remainder Theorem and Dirichlet's theorem we find that there are infinitely many primes $p$ such that $p \equiv 1\left(\bmod p_{1} p_{2}\right)$ and $p \equiv-1\left(\bmod p_{3} p_{4}\right)$ and for these primes $n=p$ satisfies $(27)$.

TheOREm 3. For all $\omega>0$ there are infinitely many $n \in \mathbb{N}$ such that

$$
d(\mathcal{A}, n)+\omega<\min (d(\mathcal{A}, n-1), d(\mathcal{A}, n+1))
$$

if and only if for all $k \in \mathbb{N}$ there exist $a_{1}, \ldots, a_{k}, b_{1}, \ldots, b_{k} \in \mathcal{A}$ so that $a_{i} \neq a_{j}$ and $b_{i} \neq b_{j}$ for $i \neq j,\left(\left[a_{1}, \ldots, a_{k}\right],\left[b_{1}, \ldots, b_{k}\right]\right) \leq 2$ and all $a_{i}, b_{j}>1$.

Proof. One direction is trivial: if (28) holds for all $\omega$ with some $n \in \mathbb{N}$ then we choose $k=[\omega]+1$, the numbers $n+1$ and $n-1$ have at least $k$ divisors $(>1)$ in $\mathcal{A}$, and these $2 k$ elements satisfy the condition.

To prove the other direction we use the Chinese Remainder Theorem and Dirichlet's theorem to deduce that there are infinitely many prime numbers $p$ for which the following two relations hold for all $i, j \in\{1, \ldots, k\}$ :

$$
\begin{aligned}
& a_{i} \mid p-1, \\
& b_{j} \mid p+1 .
\end{aligned}
$$

Now $n=p$ satisfies (28) with $\omega=k-1$, and since $k$ was an arbitrary natural number, the proof is complete.

\section{Corollaries}

Corollary 1 (Theorem of Erdős, see [1] and [3, p. 277]). For the divisor function $d(n)$, for all $k \in \mathbb{N}$ there are infinitely many $n \in \mathbb{N}$ with

$$
d(n)>\prod_{i=1}^{k} d(n-i) d(n+i) .
$$

Proof. Choose $\mathcal{A}=\mathbb{N}$ and apply Theorem 1 .

Corollary 2. For all $\omega>0$ there are infinitely many $n \in \mathbb{N}$ with

$$
d(n)+\omega<\min (d(n-1), d(n+1)) .
$$

Proof. Choose $\mathcal{A}=\mathbb{N}$ and apply Theorem 3 .

Corollary 3. For the number $\nu(n)$ of distinct prime divisors, for all $k \in \mathbb{N}$ there are infinitely many $n \in \mathbb{N}$ with

$$
\nu(n)>\prod_{i=1}^{k} \nu(n-i) \nu(n+i) .
$$

Proof. Choose $\mathcal{A}=\{p \in \mathbb{N}$ : prime $\}$ and apply Theorem 1 .

Corollary 4. For all $\omega>0$ there are infinitely many $n \in \mathbb{N}$ with

$$
\nu(n)+\omega<\min (\nu(n-1), \nu(n+1)) .
$$

Proof. Choose $\mathcal{A}=\{p \in \mathbb{N}$ : prime $\}$ and apply Theorem 3 . 
CoRollary 5. For the total number $\Omega(n)$ of prime divisors, for all $k \in \mathbb{N}$ there are infinitely many $n \in \mathbb{N}$ with

$$
\Omega(n)>\prod_{i=1}^{k} \Omega(n-i) \Omega(n+i) .
$$

Proof. Choose $\mathcal{A}=\{q \in \mathbb{N}$ : prime or power of a prime $\}$ and apply Theorem 1.

Corollary 6. For all $\omega>0$ there are infinitely many $n \in \mathbb{N}$ with

$$
\Omega(n)+\omega<\min (\Omega(n-1), \Omega(n+1)) .
$$

Proof. Choose $\mathcal{A}=\{q \in \mathbb{N}$ : prime or power of a prime $\}$ and apply Theorem 3.

Corollary 7 (Problem of Sárközy, see [5, Problem 25]). For every infinite set $\mathcal{A} \subseteq \mathbb{N}$, the sequence $|d(\mathcal{A}, n+1)-d(\mathcal{A}, n)|$ cannot be bounded.

Proof. Apply Theorem 1 for the set $\mathcal{A} \cup\{1\}$.

Corollary 8. For every infinite set $\mathcal{A} \subseteq \mathbb{N}$ and any $\omega>0$ there are infinitely many $n$ with

$$
d(\mathcal{A}, n)>\omega+\max (d(\mathcal{A}, n-1), d(\mathcal{A}, n+1)) .
$$

Proof. Apply Theorem 1 for the set $\mathcal{A} \cup\{1\}$.

Acknowledgements. András Sárközy has played an important role in the writing of this paper. I also thank him for his problem article [5]. Furthermore, I gratefully acknowledge help from Mihály Szalay.

\section{References}

[1] P. Erdős, Remarks on two problems, Mat. Lapok 11 (1960), 26-33 (in Hungarian).

[2] P. Erdős and A. Sárközy, Some asymptotic formulas on generalized divisor functions, in: Studies in Pure Mathematics, Akadémiai Kiadó, Budapest, 1983, 165-179.

[3] P. Erdős and J. Surányi, Selected Topics from Number Theory, Polygon, Szeged, 1996 (in Hungarian).

[4] H. Halberstam and H.-E. Richert, Sieve Methods, Academic Press, London, 1974.

[5] A. Sárközy, Unsolved problems in number theory, Period. Math. Hungar. 42 (2001), $17-35$.

Ronay J. u. 11

H-9026 Gyor, Hungary

E-mail: zger@bolyai1.elte.hu

Received on 8.7.2002

and in revised form on 16.1.2003 